\title{
Corneal thickness and visual field damage in glaucoma patients
}

M Papadia, C Sofianos, M lester, G Bricola, $M$ Mete and CE Traverso

\begin{abstract}
Purpose To verify whether there was a significant correlation between central corneal thickness (CCT) and visual field damage in patients with primary open angle glaucoma (POAG).

Methods A total of 99 eyes with POAG were consecutively recruited. Patients were classified as glaucomatous based on visual field and optic nerve head damage. All underwent applanation tonometry, Humphrey perimetry, and measurement of CCT with ultrasonic pachymetry. Based on CCT value, the sample was split at the mode in two groups (group $1<535 \mu \mathrm{m}, n=49$; group $2 \geq 535 \mu \mathrm{m}, n=50$ ).

Results Entire cohort: mean CCT $554 \mu \mathrm{m} \pm 45.03$; mean deviation (MD) $-6.68 \mathrm{~dB} \pm 7.32$; pattern standard deviation (PSD) 5.33 \pm 3.75 ; intraocular pressure (IOP) $17.91 \pm 4.16 \mathrm{mmHg}$ with treatment. Group 1: CCT was $504.8 \mu \mathrm{m} \pm 30.8 ; \mathrm{MD}-9.01 \mathrm{~dB} \pm 8.72$; PSD $6.38 \pm 3.99$; IOP $18.02 \mathrm{mmHg} \pm 4.66$. Group 2: mean CCT 574.6 $\mu \mathrm{m} \pm 35.03 ; \mathrm{MD}$ $-4.39 \mathrm{~dB} \pm 4.70$; PSD $4.25 \pm 3.19$; IOP $17.79 \mathrm{mmHg} \pm 3.57$. A significant difference was found between the two groups for both $\mathrm{MD}$ and PSD. Linear regression analysis showed a significant correlation between CCT and PSD $(P<0.001)$.

Conclusions Our data show that patients with a thinner cornea had a worse MD and PSD. As a thinner CCT causes an underestimation of the true IOP, there may be a delay in the diagnosis of POAG or an inadequate estimate of the clinical course despite apparently desirable IOP applanation readings.
\end{abstract}

Eye (2007) 21, 943-947; doi:10.1038/sj.eye.6702350; published online 28 April 2006

Keywords: glaucoma; corneal thickness; visual field; indices; progression

\section{Introduction}

The term glaucoma refers to a group of diseases that have in common a characteristic optic neuropathy with associated visual field loss, for which elevated intraocular pressure (IOP) is one of the primary risk factors. ${ }^{1}$ The 'normal' IOP is a statistical description of the range of IOP in the population, and is not applicable to the individual subject. ${ }^{2}$ Today, different methods to measure the IOP are available.

The most widely used instrument, considered the international gold standard, is the Goldmann applanation tonometer. Hans Goldmann applied the Imbert-Fick principle which states: 'the existing pressure in a sphere containing a liquid, whose wall is constituted by a very thin and perfectly elastic membrane, can be measured by an external compression sufficient to transform a portion of spheric surface in a plain surface'. This theoretical sphere is dry, thin-walled, and readily flexible, all features not applicable to the cornea.

The force necessary to flatten the cornea during tonometry can be influenced not only by the IOP but also by corneal characteristics such as central corneal thickness (CCT), corneal shape and hydratation, ${ }^{3}$ rigidity of the sclera and the globe.

Goldmann himself, in his first report described some of the possible sources of measurement error. ${ }^{3}$ He specifically outlined that the theoretical basis for his instrument was calculated for a mean CCT of $500 \mu \mathrm{m}$ and that the accuracy could vary if CCT was significantly different from this value.

Nowadays, CCT is considered as a possible explanation for glaucoma cases where clinical findings do not match. ${ }^{4}$ A positive correlation between IOP readings and corneal thickness was found in patients attending a general clinic. ${ }^{5}$

Recently Henderson et $a l^{6}$ demonstrated that ocular hypertension (OHT) patients with
Centro di Ricerca Clinica e Laboratorio per il Glaucoma e la Cornea, Clinica Oculistica, DiNOG, University of Genova, Azienda Ospedaliera Universitaria San Martino, Genova, Italy

Correspondence:

CE Traverso,

Clinica Oculistica Università di Genova,

Azienda Ospedaliera

Universitaria San Martino,

L.go Rosanna Benzi, 10,

16132

Genova, Italy

Tel: + 3910353 8468;

Fax: + 390103538468 .

E-mail: mc8620@

mclink.it

Received: 2 November 2005 Accepted in revised form:

7 March 2006

Published online: 28 April 2006

This study was presented in part at ARVO meeting 2005 in Ft Lauderdale, FL, USA

Declared interests: None of the authors has proprietary interest in development or marketing of any product or instrument mentioned in this article 


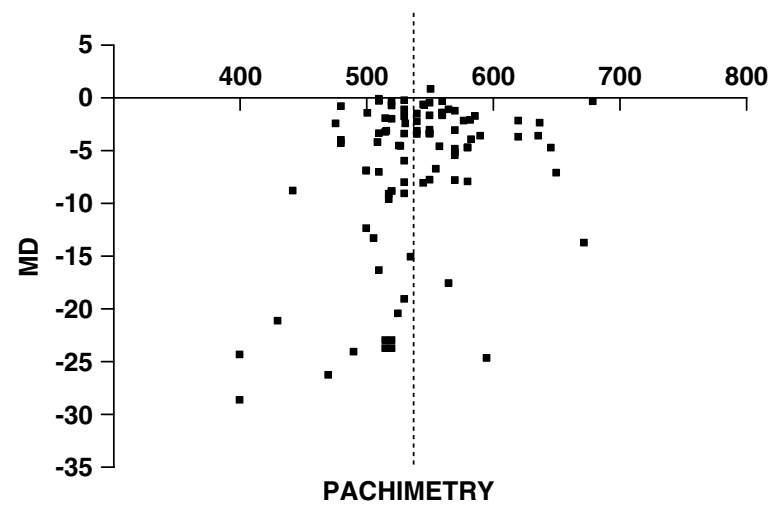

Figure 1 Scattergram of MD ( $y$-axis) and CCT ( $x$-axis). Entire group, $n=99$; the dotted line separates the two groups.

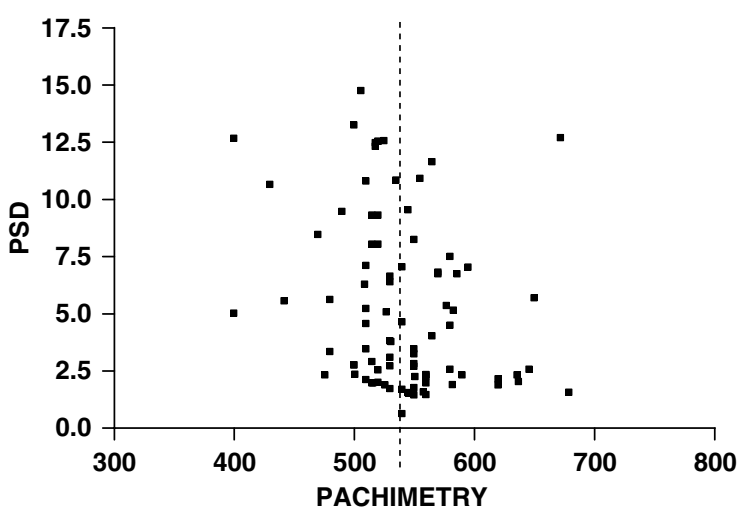

Figure 2 Scattergram of PSD ( $y$-axis) and CCT ( $x$-axis). Entire sample, $n=99$; the dotted line separates the two groups.

thinner CCT had a significant lower retinal nerve fibre layer thickness measurements compared with both OHT patients with thicker CCT and normal patients, thus suggesting that different CCT values might be associated with different probability of developing glaucoma damage.

The Ocular Hypertension Treatment Study specified some clinical and demographic factors that might represent risk factors for the development of open angle glaucoma. Among them thinner CCT was found to be a significant risk factor. ${ }^{7}$ The aim of our study was to verify whether there was a correlation between CCT and standard threshold perimetry indices in patients with primary open angle glaucoma (POAG).

\section{Materials and methods}

In total, 99 consecutive eyes with POAG treated with IOP-lowering medications for at least 5 years were prospectively recruited. All subjects were Caucasians, had a normal cornea at the slit-lamp, had no signs or history of corneal surgery or disease, and were phakic.
Visual fields were assessed by a Humphrey Field Analyzer 750 (HFA, Humphrey, Inc., San Leandro, CA, USA), 24-2 SITA (Swedish Interactive Threshold Algorithm) standard, program full threshold. Mean deviation (MD) and pattern standard deviation (PSD) were considered in this study.

Patients were classified as having POAG when a typical abnormal optic nerve head or typical damage of the visual field and open angle at gonioscopy were present.

The findings necessary to classify patients as having abnormal OHN were the optic rim notch, diffuse generalized loss of optic rim tissue, vertical cup/disc diameter ratio, asymmetry unexplained by side differences in optic disc size, and/or disc haemorrhage.

The Spaeth's gonioscopy grading system was used.

A visual field test was considered glaucomatous when abnormal Glaucoma Hemifield test was confirmed on two consecutive tests, or three abnormal points confirmed on two consecutive tests with $P<5 \%$ probability of being normal, one of which should have $P<1 \%$, all not being contiguous with the blind spot and CPSD $<5 \%$ if the visual field is otherwise normal, confirmed on two consecutive tests. ${ }^{8}$ Any defect or suspected defect had to be confirmed by repeated testing. Patients were excluded when either visual field testing was considered unreliable (false-negative and falsepositive responses $>30 \%$ and fixation losses $>20 \%$ ).

Patients were not excluded on the basis of gender, race, or age. All patients had former experience of visual field examination and all visual fields were performed by the same perimetrist. The refractive error ranged from -7 to +7 diopters.

Each patient underwent a biomicroscopic and a visual field examination, CCT and IOP measurements.

CCT was measured with ultrasonic contact pachymetry (Quantel Medical, model: POCKET, France). Pachymetry values were always obtained by an observer masked to the perimetry data. Patients were instructed to look straight ahead at a fixation target located at $3 \mathrm{~m}$. After having pushed the button to initiate corneal thickness measurements, the probe tip was gently positioned to touch the patient's cornea at its centre. The Pachymeter probe had to be perpendicular to the apex of the cornea. If the measurement was valid, a value appeared on the digital display. The mean value of three consecutive measurements was used for the statistical analysis. All measurements were taken by the same physician.

IOP was evaluated with Goldmann applanation tonometry (Haag-Streit, Switzerland).

All patients were in a sitting position and a topical anesthetic drop with fluorescein was instilled in both eyes. Each patient positioned the head on the chin-rest of 
a Haag-Streit slit-lamp biomicroscope and a Goldmann applanation measurement was performed. Patients were asked not to move their eyes, not to blink, and to continue breathing normally while looking at a target point on the slit-lamp, in order to keep the visual axis parallel to the probe.

The entire cohort of eyes was divided in two groups ranked on CCT values, separated at the mode. In group 1 , the CCT value was less than $535 \mu \mathrm{m}$, whereas in group 2 CCT value was greater than or equal to $535 \mu \mathrm{m}$.

The results were analysed by descriptive analysis and when the distribution of the data was normal, $t$-test and Pearson's $r$ coefficient were used to compare and correlate CCT, MD, and PSD. When the distribution of the data was non-normal, Mann-Whitney test and Spearman coefficient were used. A linear regression analysis was used.

The IRB approved the study without the need for a specific informed consent as pachymetry is noninvasive and considered as a routine part of a tertiary care glaucoma evaluation.

\section{Results}

The mean refractive error of the included patients was $-1.7 \pm 4.7$ (mean $\pm \mathrm{SD}$ ) diopters and the mean age was $63 \pm 16$ years. The mean value of CCT for the entire sample was $554 \mu \mathrm{m} \pm 45$; MD, $-6.68 \pm 7.32 \mathrm{~dB}$, PSD $5.33 \pm 3.75$, and IOP $17.9 \pm 4.16 \mathrm{mmHg}$ with treatment.

Figure 1 shows the Scattergram of MD (y-axis) and CCT (x-axis); Figure 2 shows the Scattergram of PSD (y-axis) and CCT (x-axis).

Table 1 Correlation between CCT and other parameters

\begin{tabular}{lrc}
\hline & \multicolumn{1}{c}{$\mathrm{r}^{2}$} & P-value \\
\hline MD & 0.21 & 0.039 \\
PSD & -0.29 & 0.006 \\
IOP & 0.09 & 0.40 \\
\hline
\end{tabular}

$\mathrm{CCT}$, central corneal thickness; IOP, intraocular pressure; MD, mean deviation; PSD, pattern standard deviation.

Entire sample, $n=99$.

Table 1 shows the correlation between CCT and the other parameters of the entire sample calculated with linear regression.

Table 2 shows the comparison between group 1 $(n=49)$ and group $2(n=50)$. The mean MD value for the group 1 was significantly lower $(P<0.001)$ compared to the mean MD value of group $2(-9.01 \pm 8.72$ and $-4.39 \pm 4.70 \mathrm{~dB}$, respectively).

The mean PSD value for the group 1 was significantly higher $(P<0.05)$ than the mean PSD value of group 2 $(6.38 \pm 3.99$ and $4.25 \pm 3.19$, respectively)

No differences were found in the IOP between the two groups.

Table 3 shows the linear regression analysis of age vs MD or PSD for the entire sample and for the two groups. No correlation was found among these parameters.

The refractive error was $-2.1 \pm 5.2$ diopters in group 1 and $-1.3 \pm 4.2$ diopters in group 2 .

\section{Discussion}

In 1975, Ehlers measured the 'real' or 'manometric' IOP using a cannulation method in a group of 29 normal eyes undergoing cataract surgery and correlated corneal thickness to measurement errors of Goldmann applanation tonometry. ${ }^{9}$ The IOP value measured with

Table 3 Linear regression of age vs MD or PSD, both in the entire sample and in the two groups

\begin{tabular}{ccc}
\hline Group & $P S D$ & $M D$ \\
\hline $\begin{array}{c}\text { Entire } \\
r^{2}\end{array}$ & 0.004 & 0.002 \\
$P$ & 0.695 & 0.822 \\
& & \\
Thin & & \\
$r^{2}$ & 0.022 & 0.044 \\
$P$ & 0.53 & 0.40 \\
& & \\
Thick & & 0.080 \\
$r^{2}$ & 0.154 & 0.328 \\
$P$ & 0.146 & \\
\hline MD, mean deviation; PSD, pattern standard deviation.
\end{tabular}

Table 2 Comparison between group $1(n=49)$ and group $2(n=50)$

\begin{tabular}{|c|c|c|c|c|c|}
\hline & \multicolumn{2}{|c|}{ Group $1(<535 \mu \mathrm{m}) \mathrm{n}=49$} & \multicolumn{2}{|c|}{ Group $2(>535 \mu m) \mathrm{n}=50$} & \multirow{2}{*}{$\frac{\mathrm{t} \text {-test }}{\text { P-value }}$} \\
\hline & Mean & $S D$ & Mean & $S D$ & \\
\hline $\mathrm{CCT}(\mu \mathrm{m})$ & 504.8 & 30.8 & 574.6 & 35.03 & $<0.001$ \\
\hline MD (dB) & -9.01 & 8.72 & -4.39 & 4.70 & 0.0014 \\
\hline PSD (dB) & 6.38 & 3.99 & 4.25 & 3.19 & 0.0076 \\
\hline IOP (mm Hg) & 18.02 & 4.66 & 17.79 & 3.57 & 0.957 \\
\hline
\end{tabular}

CCT, central corneal thickness; IOP, intraocular pressure; MD, mean deviation; PSD, pattern standard deviation. 
Goldmann tonometer was equal to the manometric one when CCT value was about $520 \mu \mathrm{m}$. Deviations from this value resulted in overestimation or underestimation of IOP that could be calculated as $7 \mathrm{mmHg}$ every $100 \mu \mathrm{m}$ of thickness; CCT was significantly correlated with the IOP measured by applanation tonometry, but no correlation was found between the IOP measured with applanation tonometry and the corneal radius. In 1978, Johnson et al ${ }^{10}$ published the data of a patient with CCT of $900 \mu \mathrm{m}$ that despite hypotonic therapy did not lower its IOP In 1991, Graef $^{11}$ demonstrated that different CCT caused different readings of IOP both with applanation tonometry and Reichert noncontact tonometry (NCT II). In 2004, Brandt et $a l^{12}$ published data suggesting that CCT measurements may be useful in the management of glaucomatous patients. Nowadays, many studies have demonstrated that CCT vary significantly in the general population and that this difference may cause misclassification of patients whenever Goldmann applanation tonometry IOP value is used for diagnostic definitions. ${ }^{9-14}$ There is general agreement on the finding that patients with ocular hypertension have thicker central corneas, ${ }^{15,16}$ whereas cases of normal pressure glaucoma have thinner than average central corneas. ${ }^{17,18}$ The relationship between pachymetry values and the risk of glaucoma damage is still controversial. No correlation between the thickness of the central cornea, of the peripapillary retinal nerve fibres, ${ }^{19}$ and of the lamina cribrosa ${ }^{20}$ was found in nonglaucomatous human eyes; it is not known whether hystomorphometry of the lamina cribrosa or peripapillary nerve fibre layer thickness in glaucomatous eyes would show a relationship with corneal thickness. There is no consensus on the influence of pachymetry values on the likelihood of progression of glaucomatous damage in established glaucoma. Kim and $\mathrm{Chen}^{21}$ and Herndon $e t \mathrm{al}^{22}$ proved the association of thinner central cornea values with VF progression in glaucoma patients.

Jonas et $a l^{23}$ and Chauhan et $a l^{24}$ found an association among lower CCT and worse base line VF, but the lower CCT was not associated with progression of glaucomatous optic nerve neuropathy.

In our study, a significant correlation was found between CCT and both MD and PSD, with thinner corneas significantly associated with worse damage. When we analysed the correlation in the two groups, the significance was less for PSD probably because of its nonlinear behaviour, for the $50 \%$ reduction of the number of eyes in the each group, or for the different distribution of the values. When the entire cohort was divided into two groups on the basis of CCT, a significant difference was found for both MD and PSD. Ocular hypertensives and POAG cases with very early damage were not included. Our data suggest that a thinner cornea, causing a systematic underestimation of the true IOP, could very well lead to a late diagnosis and as a consequence to greater progression of damage despite apparently 'safe' treated IOP. On the other hand, patients with a thick cornea despite a possibly lower risk of progression are more likely to be treated.

Our data did not show any correlation between CCT and IOP probably because all patients were already treated with IOP-lowering medications; this type of correlation was outside the purpose of this study.

In our study, no correlation was found between age and CCT and between age and VF indices.

It is assumed that CCT does not change during adult life. Whether this applies to glaucoma patients undergoing chronic topical treatment is not known yet.

A simple exam as pachymetry might be useful to obtain a prognostic factor for the onset of glaucoma or for the severity of glaucomatous damage. Thin central corneas were shown to be a strong factor associated with progression from $\mathrm{OH}$ to $\mathrm{POAG}^{7,16,25}$ or for the appearance of early defects with nonconventional perimetry in OHTs. ${ }^{26,27}$

In summary, our data show a definite inverse relationship between the central corneal pachymetry value and the damage of the visual field.

Should the correlation between corneal thickness and progression of disease be confirmed longitudinally for POAG, the pachymetry value could become helpful also for cross-sectional evaluations even without an IOPcorrecting algorithm.

Our study has limitations. It was of sectional nature and therefore does not allow direct estimation of the influence of CCT on the progression of disease.

Study selection criteria limited the sample to patients with unquestionable glaucoma damage, not yielding data on glaucoma suspects or ocular hypertensives.

Our data support the existence of a de facto relationship between CCT and stage of glaucoma damage. Whether this is related to IOP-measuring error or to intrinsic ocular factor associated with thinner CCT remains to be assessed and warrants further studies.

\section{References}

1 American Academy of Ophthalmology. Basic and Clinical Science Course (BCSC) Section 10 'Glaucoma', American Academy of Ophthalmology edition, Vol. 1 American Academy of Ophthalmology: San Francisco, CA, USA, 2002-2003 pp 5-13.

2 European Glaucoma Society. Terminology and Guidelines for Glaucoma, European Glaucoma Society IInd edn, Vol 1.1 Dogma: Savona, 2003 pp 1-3.

3 Goldmann H, Schmidt T. Ueber applanationtonometrie. Ophthalmologica 1957; 134: 221-242.

4 European Glaucoma Society. Terminology and Guidelines for Glaucoma, European Glaucoma Society. 2nd edn., Vol. 2.2 Dogma: Savona, 2003 pp 2-5. 
5 Shah S, Chatterjee A, Mathai M, Kelly SP, Kwartz J, Henson D et al. Relationship between corneal thickness and measured intraocular pressure in a general ophthalmology clinic. Ophthalmology 106: 2154-2160.

6 Henderson PA, Medeiros FA, Zangwill LM, Weinreb RN. Relationship between central corneal thickness and retinal fiber layer thickness in ocular hypertensive patients. Ophthalmology 2005; 112: 251-256.

7 Kass MA, Heuer DK, Higginbotham EJ, Johnson CA, Keltner JL, Miller JP et al. The Ocular Hypertension Treatment Study: a randomized trial determines that topical ocular hypotensive medication delays or prevents the onset of primary open-angle glaucoma. Arch Ophthalmol 2002; 120: 701-713.

8 European Glaucoma Society. Terminology and Guidelines for Glaucoma, European Glaucoma Society IInd ed., Vol. 1.1 Dogma: Savona, 2003 pp 1-27.

9 Ehlers N, Bramsen T, Sperling S. Applanation tonometry and central corneal thickness. Acta Ophthalmol (Copenh) 1975; 53: 34-43.

10 Johnson M, Kass MA, Moses RA, Grodzki WJ. Increased corneal thickness simulating elevated intraocular pressure. Arch Ophthalmol 1978; 96: 664-665.

11 Graef M. Zur Bedeutung der Hornhautdicke bei der non-contact-tonometrie. Kli Mbl Augenheilk 1991; 199: 183-186.

12 Brandt JD, Beiser JA, Gordon MO, Kass MA. The ocular hypertension treatment study (OHTS) group. Central corneal thickness and measured IOP response to topical ocular hypotensive medication in the ocular hypertension study. Am J Ophthalmol 2004; 138: 717-722.

13 Whitacre MM, Stein R. Sources of error with Goldmanntype tonometres. Surv Ophthalmol 1993; 38: 1-30.

14 Whitacre MM, Stein R, Hassanein K. The effects of corneal thickness on applanation tonometry. Ophthalmology 1993; 115: 592-596.

15 Argus WA. Ocular hypertension and central corneal thickness and curvature in normal tension glaucoma. Am J Ophthalmol 1995; 102: 1810-1812.
16 Zeppieri M, Brusini P, Miglior S. Corneal thickness and functional damage in patients with ocular hypertension. Eur J Ophthalmol 2005; 15: 196-201.

17 Morad Y, Sharon E, Hefetz L, Nemet P. Corneal thickness and curvature in normal tension glaucoma. Am J Ophthalmol 1998; 125: 164-168.

18 Emara BY, Tingey DP, Probst LE, Motolko MA. Central corneal thickness in low-tension glaucoma. Can J Ophthalmol 1999; 34: 319-324.

19 Iester M, Mermoud A. Retinal nerve fiber layer and physiological central corneal thickness. J Glaucoma 2001; 10: $158-162$.

20 Jonas JB, Holbach L. Central corneal thickness and thickness of the lamina cribrosa in human eyes. Invest Ophthalmol Vis Sci 2005; 46: 1275-1279.

21 Kim WJ, Chen PP. Central corneal pachymetry and visual field progression in patients with open-angle glaucoma. Ophthalmology 2004; 111: 2126-2132.

22 Herndon LW, Weizer JS, Stinnett SS. Central corneal thickness as a risk factor for advanced glaucoma damage. Arch Ophthalmol 2004; 122: 17-21.

23 Jonas JB, Stroux A, Velten I, Juenemann A, Martus P, Budde WM. Central corneal thickness correlated with glaucoma damage and rate of progression. Invest Opthalmol Vis Sci 2005; 46: 1269-1274.

24 Chauhan BC, Hutchison DM, LeBlanc RP, Artes PH, Nicolela MT. Central corneal thickness and progression of the visual field and optic disc in glaucoma. Br J Ophthalmol 2005; 89: 1008-1012.

25 Brusini P, Tosoni C, Parisi L, Rizzi L. Ocular hypertension and corneal thickness: a long-term prospective study. Results after two years. Eur J Ophthalmol 2005; 15: 550-555.

26 Medeiros FA, Sample PA, Weinreb RN. Corneal thickness measurements and visual function abnormalities in ocular hypertensive patients. Am J Ophthalmol 2003; 135: 131-137.

27 Medeiros FA, Sample PA, Zangwill LM, Bowd C, Aihara M, Weinreb RN. Corneal thickness as a risk factor for visual field loss in patients with preperimetric glaucomatous optic neuropathy. Am J Ophthalmol 2003; 136: 805-813. 